\title{
TTR
}

Traduction, terminologie, rédaction

\section{Daniel Weissbort et Astradur Eysteinsson, dirs. Translation. Theory and Practice. A Historical Reader. Oxford et New York, Oxford University Press, 2006, xiv + 650 p.}

\section{Marco A. Fiola}

Volume 22, numéro 1, 1er semestre 2009

La traduction au Japon

Translation in Japan

URI : https://id.erudit.org/iderudit/044789ar

DOI : https://doi.org/10.7202/044789ar

Aller au sommaire du numéro

Éditeur(s)

Association canadienne de traductologie

ISSN

0835-8443 (imprimé)

1708-2188 (numérique)

Découvrir la revue

Citer ce compte rendu

Fiola, M. A. (2009). Compte rendu de [Daniel Weissbort et Astradur Eysteinsson, dirs. Translation. Theory and Practice. A Historical Reader. Oxford et New York, Oxford University Press, 2006, xiv + 650 p.] TTR, 22(1), 257-260

https://doi.org/10.7202/044789ar

Tous droits réservés (C) TTR: traduction, terminologie, rédaction — Les auteurs, 2010
Ce document est protégé par la loi sur le droit d'auteur. L'utilisation des services d'Érudit (y compris la reproduction) est assujettie à sa politique d'utilisation que vous pouvez consulter en ligne.

https://apropos.erudit.org/fr/usagers/politique-dutilisation/ 


\section{COMPTES RENDUS}

\section{Daniel Weissbort et Astradur Eysteinsson, dirs. Translation. Theory and Practice. A Historical Reader. Oxford et New York, Oxford University Press, 2006, xiv +650 p.}

De prime abord, l'ouvrage que nous proposent Weissbort et Eysteinsson impressionne par son ampleur et sa richesse. Les auteurs ont en effet rassemblé une anthologie aux visées pédagogiques qui répondra sans aucun doute aux attentes des spécialistes de la littérature comparée, et peut-être même à d'autres. En effet, même si l'ouvrage est destiné d'abord et avant tout aux comparatistes, les auteurs nont pas hésité pour autant à puiser à la source de quelques traductologues ouvrant hors des sentiers littéraires. Cependant, si l'on devait faire un reproche à l'ouvrage, ce serait d'être en grande partie eurocentriste, voire anglocentriste, hormis pour quelques textes de la première partie portant sur la traduction à une époque antérieure à l'apparition de la langue anglaise et une ou deux incursions du côté des langues asiatiques dans la dernière partie. Par ailleurs, l'ouvrage accorde une place de choix aux chercheurs modernes et contemporains, même si, à notre avis, plusieurs noms brillent par leur absence. On s'explique mal ces lacunes par le simple fait que les corpus et les auteurs retenus sont issus principalement de la traduction littéraire car, si le texte pragmatique ne jouit pas du même prestige que le texte littéraire, il nen demeure pas moins que, chez les contemporains, d'aucuns s'intéressant essentiellement à la traduction pragmatique ont apporté à la recherche des contributions remarquables dont peuvent bénéficier les traducteurs littéraires. Discipline transdisciplinaire par excellence, la traductologie ne devrait plus souffrir ce type de cloisonnement arbitraire.

En ce qui concerne la structure de louvrage, on trouve deux grandes parties, la première, intitulée From Antiquity to Modern Times, suivant une périodisation allant de Cicéron à Caxton, d'une part, jusqu'au XIX ${ }^{e}$ siècle, d'autre part, en passant par l'époque de la Réformation et de la Renaissance. La seconde grande partie, quant à elle, se divise en deux sous-sections, soit From Pound to Nabokov, puis Recent and Contemporary Writings. 
Tout en étant le plus bref, le chapitre premier couvre de loin la plus grande tranche d'histoire. Par conséquent, c'est par l'ampleur de la période plutôt que par la profondeur d'analyse que se caractérise le chapitre. Ce traitement peut s'expliquer également en partie par le fait que le chapitre s'intéresse sans contredit à la traduction comme phénomène littéraire dans le monde anglophone. La plupart des écrits-phares sur la traduction (par Cicéron, Horace et Jérôme), qui sont cités ultérieurement dans louvrage, de la Renaissance au XIX ${ }^{\mathrm{e}}$ siècle, sont également inclus dans cette partie. Caractéristique des recueils historiques, l'ouvrage comprend également un certain nombre d'anecdotes mémorables, comme celle de la traduction du Tanakh qui a mené à la Septante. D'ailleurs, dans ce premier chapitre, la traduction des Écritures occupe une place de choix.

Le chapitre 2, From the Reformation and the Renaissance to the Eighteenth Century, s'intéresse à la traduction de la Bible en Angleterre, incluant un commentaire de Martin Luther sur la traduction. En dépit du grand intérêt que cette période comporte pour l'étude de la traduction littéraire, y compris la traduction biblique, les auteurs n'ont pas jugé opportun d'aller au-delà du phénomène de la traduction biblique dans la langue du peuple pour se pencher sur l'importance du rôle de la traduction dans le mouvement de la Réforme, c'est-à-dire sur tous les " problèmes » de traduction qui ont donné lieu à des interprétations nouvelles et ouvert la porte à de nouvelles manières d'interpréter les Écritures. Ce chapitre accorde également une place de choix à plusieurs traducteurs de renom, y compris Dryden, Pope et Johnson. On note au passage quelques sections sur la traduction en anglais d'auteurs français, plus particulièrement Étienne Dolet, Joachim du Bellay et Anne Dacier. D'ailleurs, le chapitre contient une section sur les traductrices de l'époque.

Plus on s'approche de l'époque contemporaine, et plus le contenu des chapitres est éclectique. En effet, le chapitre 3 traite de traducteurs du XIX ${ }^{\mathrm{e}}$ tels que Longfellow, Browning, Morris et Rossetti, en plus de consacrer bon nombre de pages aux incontournables Goethe et Schleiermacher, sans oublier quelques critiques de traductions parues à l'époque victorienne. On comprend certes pourquoi Goethe et Schleiermacher trouvent 
leur place dans ce chapitre, mais on s'explique mal l'absence d'autres figures non moins marquantes de la traduction, comme Humboldt et Hölderlin, mises de côté bien évidemment pour des raisons autres que le fait qu'ils n'aient pas traduit à partir de, ou vers, l'anglais.

C'est au chapitre 4, From Pound to Nabokor, que l'on trouve les premières références substantielles à la traduction d'œuvres romanesques, et c'est à partir de là que l'on reconnaît le problème du genre textuel, problème trop souvent ignoré au profit d'autres angles d'étude comme le registre, le lexique et les caractéristiques des auteurs. Tout lecteur un tant soit peu averti ne manquera pas de noter certaines lacunes à l'égard de l'œuvre de Pound traducteur, notamment ses traductions du chinois. Cet oubli ne peut qu'alimenter les critiques concernant le penchant anglocentriste de l'ouvrage et l'exclusion quasi systématique de la traduction de langues non européennes. La seconde partie du chapitre 4 traite de plusieurs auteurs qui ont écrit également sur la traduction, notamment Benjamin, Levý et Borges, pour finalement se terminer sur une longue section réservée à Nabokov, (auto)traducteur controversé et auteur du non moins controversé Art of Translation. Cette partie se conclut par une brève section sur sa traduction de Eugene Onegin.

Le chapitre 5, qui ne constitue pas moins du tiers de l'ouvrage, accorde une place de choix aux théoriciens de la traduction, les traducteurs se trouvant relégués au second plan. Bien entendu, le chapitre est loin d'être exhaustif, et d'aucuns déploreront de grands oubliés. Pour notre part, il nous semble qu'il aurait été juste d'inclure à ce chapitre une ou deux entrées consacrées à l'interprétation, pratique et profession qui a certes précédé la traduction dans l'histoire et qui fournit un poste d'observation intéressant pour l'étude de la traduction. Toutefois, étant donné que les chapitres précédents se sont surtout intéressés à la traduction comme forme d'expression écrite, on ne sétonnera pas de l'absence de mentions relatives aux chercheurs qui considèrent la traduction à travers le prisme de l'interprétation. On saluera au passage l'inclusion de certains traducteurs peu connus, mais on s'expliquera toutefois assez mal l'exclusion d'autres traductologues qui auraient pu être pris en compte, 
notamment Baker et Newmark, pour ne nommer que ceux-là. Il faut parcourir la bibliographie de lectures supplémentaires pour trouver quelque référence à ces auteurs. D'autres, comme Catford, Reiss, Mounin, sont certes mentionnés, mais seulement au passage. C'est une faiblesse de l'ouvrage, surtout lorsqu'on pense au titre, qui laisse présager bien davantage.

On trouve en fin d'ouvrage un index des noms propres fort utile. On regrettera toutefois l'étendue de la bibliographie des lectures supplémentaires, divisée en trois parties : Journals; Encyclopedias, Anthologies, Essay Collections; General or Introductory Books on the Practice of Translation and/or the Field of Translation Studies. En plus d'être succincte, cette bibliographie semble s'être arrêtée avec le siècle précédent. En effet, on note seulement deux entrées datant de 2005, une de 2004, deux de 2003, quatre de 2002, cinq de 2001, et trois de 2000. Bien entendu, la visée historique de l'ouvrage peut permettre d'expliquer le faible nombre d'ouvrages de la plume de chercheurs contemporains. Ainsi, la jeune chercheuse qui voudra être au fait des études traductologiques devra aller puiser ailleurs les nombreux compléments d'information qui lui seront nécessaires à la compréhension de notre champ d'étude.

\title{
Marco A. Fiola UNIVERSITÉ RYERSON
}

\begin{abstract}
Daniel Blampain, Philippe Thoiron et Marc Van Campenhoudt (dirs). Mots, termes et contextes. Actes des septièmes Journées scientifiques du réseau de chercheurs Lexicologie, terminologie et traduction. Paris, Contemporary Publishing International Éditions des archives contemporaines - Agence universitaire de la Francophonie, 2006, 741 p.
\end{abstract}

Dans leur pratique quotidienne, les terminologues, traducteurs, lexicographes et lexicologues étudient, consultent, décortiquent et analysent les contextes dont ils ont besoin pour élaborer le système conceptuel d'un domaine, rendre de façon efficace un texte dans une langue différente, choisir une citation pertinente ou encore (re)définir des mots. La problématique des contextes en linguistique de corpus n'est pas nouvelle, mais elle gagne en 